\title{
AN EVOLUTIONS EQUATION AS THE WKB CORRECTION IN LONG-TIME ASYMPTOTICS OF SCHRÖDINGER DYNAMICS
}

\author{
SERGEY A. DENISOV
}

\begin{abstract}
We consider the 3-dimensional Schrödinger operator with slowly decaying potential whose radial derivatives are short-range. The long-time asymptotics for solution of the corresponding non-stationary equation is established. In this case, the standard WKB-correction should be replaced by the solution of certain evolution equation.
\end{abstract}

Consider the Schrödinger operator

$$
H=-\Delta+V, \quad x \in \mathbb{R}^{3}
$$

In this paper, we assume that potential $V(x)$ satisfies the following conditions:

\section{Conditions A:}

$$
|V|<C r^{-\gamma},\left|\frac{\partial V}{\partial r}\right|<C r^{-1-\gamma},\left|\frac{\partial^{2} V}{\partial r^{2}}\right|<C r^{-1-2 \gamma}, V(x) \in C^{2}\left(\mathbb{R}^{3}\right), \quad r=|x|
$$

and

$$
1>\gamma>1 / 2
$$

We will study the scattering properties of $H$, in particular the long-time asymptotics of the group $e^{i t H}$. This group gives the solution to the non-stationary equation: if $\psi(t)=e^{i t H} f$ and $f \in L^{2}\left(\mathbb{R}^{3}\right)$, then

$$
\partial \psi / \partial t=i H \psi, \quad \psi(0)=f
$$

The scattering theory for Schrödinger operator is a classical subject (see, e.g. 14, [15, 5]). We want to mention here several results that motivated us to write this note. Some of them are rather old and well-known, some are quite new.

The following theorem can be found in [14] (Theorem 4.1), 16] (see also [7, 1, 6] for other results on long-range scattering).

Theorem 0.1. (Yafaev [14, 16]) Assume that

$$
\left|D^{k} V(x)\right|<C\langle x\rangle^{-\gamma-|k|}
$$

with $\gamma>1 / 2$ and any $k:|k|<k_{0}, k_{0}$-big enough. Introduce

$$
\Xi(x, t)=|x|^{2} /(4 t)-t \int_{0}^{1} V(s x) d s
$$

THIS IS A PREPRINT OF AN ARTICLE WHOSE FINAL AND DEFINITIVE FORM HAS BEEN PUBLISHED IN COMM. PARTIAL DIFFERENTIAL EQUATIONS, COPYRIGHT OF TAYLOR AND FRANCIS, AVAILABLE ONLINE AT HTTP://WWW.INFORMAWORLD.COM 
and

$$
\mathcal{E}_{0}(t)[f](x)=\exp [i \Xi(x, t)](2 i t)^{-3 / 2} \hat{f}(x /(2 t))
$$

where $\hat{f}$ denotes the Fourier transform of $f$. Then, the limits

$$
\mathcal{W}^{ \pm}=s-\lim _{t \rightarrow \pm \infty} \exp (i H t) \mathcal{E}_{0}(t)
$$

exist. They are called the modified wave operators 1 .

Note that the modification is done in the physical space by the integral of potential over the segments $\{t x, t \in[0,1]\}$. We have

$$
\exp [i \Xi(x, t)]=\exp \left[i|x|^{2} /(4 t)\right] \cdot \exp \left[-i t \int_{0}^{1} V(s x) d s\right]
$$

and the second factor will be called the WKB-correction in the long-time asymptotics of $\exp (i t H)$. For the short-range case (i.e., when $\gamma>1$ ) this factor can be discarded. Perhaps, the definition of wave (modified wave) operators in momentum space is more standard (see, e.g. [14, 8]). Nevertheless, in our paper, we will be working in the physical space only.

The existence of modified wave operators per se does not imply that the spectrum of $H$ is purely absolutely continuous on positive half-line. Therefore, the following result (see, e.g., 14, Theorem 4.2 and references therein) complements the Theorem 0.1

Theorem 0.2. Assume that $V(x)$ is such that

$$
\left|\frac{\partial V}{\partial r}\right|<C r^{-1-}
$$

Then, the positive spectrum of $H$ is absolutely continuous.

The proof of this result is based on the Mourre estimates and also shows that the absorption principle holds in the suitable weighted spaces. It also allows to handle the short-range perturbations. Other proofs (see, e.g., [12, 10]) are using various PDE techniques and can handle more general cases.

In the meantime, we are not aware of any results where the existence of modified wave operators is proved in the case when the strong decay is assumed only for the radial derivative of the potential and nothing is known about the tangential component of the gradient. If, instead, the tangential component of the gradient is decaying fast, then the recent result by Perelman [11] shows that the a.c. spectrum of $H$ fills $\mathbb{R}^{+}$but it can coexist with singular spectrum. In [1], the WKB correction for the spatial asymptotics of Green's function is proved to be similar to that in the Theorem 0.1] the modification comes from the integration of potential over the segments. In papers 2, 9, the scattering theory for Hamiltonians with zero-degree homogeneous potentials was considered. The dynamics in this case is nontrivial and the analysis is quite intricate.

In this paper, we consider potential that satisfies Conditions A. The Theorem 0.2 guarantees that the positive spectrum is purely absolutely continuous. Our goal is to obtain an analog of Theorem 0.1 In our opinion, the most interesting part of this paper is new WKB correction to the asymptotic: 2 . This correction is not so

\footnotetext{
${ }^{1}$ Actually, any $\gamma \in(0,1)$ was treated but the modification $\Xi$ is then more involved

${ }^{2} \mathrm{C}$. Gérard kindly mentioned to us that somewhat similar corrections were obtained in Sections 3.6 and 4.8 of $[5]$.
} 
surprising in view of recent calculations done for sparse slowly decaying potentials (see discussion in [3]).

The paper is divided into two parts. In the first one, we introduce and study the evolution equation which gives the right WKB correction. The second part contains the proof of the main Theorem, several corollaries and examples. We will use the following notations: $\langle x\rangle$ stands for $\left(|x|^{2}+1\right)^{1 / 2}, \mathcal{F}$ denotes the Fourier transform normalized to be unitary map from $L^{2}\left(\mathbb{R}^{3}\right)$ onto itself, the symbol $D$ denotes the differential of a function, $\Sigma$ stands for the two-dimensional unit sphere, $B$ denotes the (positive) Laplace-Beltrami operator on $\Sigma$. The symbols $H^{\alpha}(\Sigma)$ stand for Sobolev spaces with index $\alpha$. For any vector $x \neq 0, x \in \mathbb{R}^{3}$, we write $\hat{x}=x /|x|$. For smooth function $f(x), x \in \mathbb{R}^{3}, f_{r}$ will mean the radial derivative and $r=|x|$. Symbol $\mathcal{D}(A)$ stands for the domain of definition of self-adjoint operator $A$ and $E_{\lambda}(A)$ is its orthoprojector.

Acknowledgement. We are grateful to A. Ionescu and A. Kiselev for useful discussions and to C. Gérard, I. Herbst, and D. Yafaev for providing us with some very important references.

\section{Evolution EQuation}

In this section, we discuss one very special evolution equation which will play an important role later. It can be easily studied by well-known methods but we will give all details for completeness.

Consider the following evolution equation:

$$
i k y_{\tau}(\tau, \theta)=\frac{(B y)(\tau, \theta)}{\tau^{2}}+V(\tau, \theta) y(\tau, \theta), \tau>0
$$

where $k \in \mathbb{R} \backslash\{0\}, V(\tau, \theta)$ is real-valued, and the function $y(\tau, \theta) \in L^{2}(\Sigma)$ for any $\tau>0$. Later on, we will let $V(\tau, \theta)=V(\tau \cdot \theta)$ where the potential $V(x)$ is the Schrödinger potential introduced before. Let us define and study $U\left(k, \tau_{0}, \tau\right) f$ - the solution of (6) satisfying an initial condition $U\left(k, \tau_{0}, \tau_{0}\right) f=f$ where $\tau, \tau_{0}>0$ and $f \in L^{2}(\Sigma)$. Note first that if $V=0$, then we can write the solution in terms of more familiar Schrödinger evolution on the sphere:

$$
U_{0}\left(k, \tau_{0}, \tau\right)=\exp \left[\frac{1}{i k} \frac{\tau-\tau_{0}}{\tau \tau_{0}} B\right]
$$

For any fixed $f \in L^{2}(\Sigma)$, the following limit exists

$$
\lim _{\tau \rightarrow \infty} U_{0}\left(k, \tau_{0}, \tau\right) f=\exp \left[\frac{1}{i k} \frac{B}{\tau_{0}}\right] f
$$

Then, $U\left(k, \tau_{0}, \tau\right)$ allows the formal Duhamel expansion involving $U_{0}(k, s, t)$ and $V(s)$. Assuming $\|V(\tau, \cdot)\|_{L^{\infty}(\Sigma)} \in L_{\text {loc }}^{1}\left(\mathbb{R}^{+}\right)$one can easily prove convergence of this expansion in $L^{2}(\Sigma)$ and we define $U\left(k, \tau_{0}, \tau\right)$ in this way. For simplicity, from now on we assume that $V \in C^{2}((0, \infty) \times \Sigma)$. Then, for $f \in H^{2}(\Sigma), U\left(k, \tau_{0}, \tau\right) f$ gives the actual solution to differential equation. Notice that $U\left(k, \tau_{0}, \tau\right)$ is unitary on $L^{2}(\Sigma)$ and $U^{*}\left(k, \tau_{0}, \tau\right)=U^{-1}\left(k, \tau_{0}, \tau\right)=U\left(k, \tau, \tau_{0}\right)$. This is an elementary property of any evolution equation with self-adjoint coefficients. If $V(\tau, \theta)$ decays fast enough, then the perturbation theory easily yields existence of the strong limit for $U\left(k, \tau_{0}, \tau\right)$ as $\tau \rightarrow+\infty$. For instance, we have the following elementary Lemma 
Lemma 1.1. If $\|V(\tau, \cdot)\|_{\infty} \in L^{1}[1, \infty)$, the the limit

$$
\lim _{\tau \rightarrow \infty} U\left(k, \tau_{0}, \tau\right) f=U\left(k, \tau_{0}, \infty\right) f
$$

exists for any $f \in L^{2}(\Sigma)$.

Proof. If $U\left(k, \tau_{0}, \tau\right)=U_{0}\left(k, \tau_{0}, \tau\right) Y(\tau)$, then

$$
Y_{\tau}(\tau)=U_{0}\left(k, \tau_{0}, \tau\right)^{-1} V(\tau) U\left(k, \tau_{0}, \tau\right) Y(\tau)
$$

That yields $\|Y\|$ is bounded in $\tau$. Since $\left\|Y_{\tau}\right\| \in L^{1}[1, \infty)$, the Cauchy criteria guarantees that $Y(\tau)$ converges to $Y(\infty)$ in norm topology. $U_{0}\left(k, \tau_{0}, \tau\right)$ converges to $U_{0}\left(k, \tau_{0}, \infty\right)$ strongly and so we get the statement of the Lemma.

Consider the following conditions that are essentially identical to Conditions A:

Conditions A1:

$\|V(\tau, \cdot)\|_{L^{\infty}(\Sigma)}<C \tau^{-\gamma},\left\|\frac{\partial V}{\partial \tau}(\tau, \cdot)\right\|_{L^{\infty}(\Sigma)}<C \tau^{-1-\gamma},\left\|\frac{\partial^{2} V}{\partial \tau^{2}}(\tau, \cdot)\right\|_{L^{\infty}(\Sigma)}<C \tau^{-1-2 \gamma}$

and

$$
V(\tau, \theta) \in C^{2}([1, \infty) \times \Sigma), \tau \geq 1,1>\gamma>1 / 2
$$

As we will see later, the solution to evolution equation does not have to have any limit as $\tau \rightarrow \infty$ in this case. In the meantime, one can obtain some estimates on the decay of various derivatives. From now on, the norm $\|\cdot\|$ means $L^{2}(\Sigma)$ norm. For any $f \in L^{2}(\Sigma)$, denote

$$
W(k, \tau) f=U(k, 1, \tau) f
$$

Lemma 1.2. Assume that $V$ satisfies Conditions $A 1$ and $k \in I \subset \mathbb{R}^{+}, f \in H^{4}(\Sigma)$. Then, the following estimates hold true

$$
\begin{aligned}
\left\|W_{\tau}(k, \tau) f\right\| & <C \tau^{-\gamma} \\
\left\|W_{\tau \tau}(k, \tau) f\right\| & <C \tau^{-2 \gamma} \\
\left\|W_{k}(k, \tau) f\right\| & <C \tau^{1-\gamma} \\
\left\|W_{\tau k}(k, \tau) f\right\| & <C \tau^{1-2 \gamma} \\
\left\|W_{k k}(k, \tau) f\right\| & <C \tau^{2-2 \gamma}
\end{aligned}
$$

where the constant $C$ depends on $I,\|f\|_{H^{4}(\Sigma)}$, and constants in the Conditions A1.

Proof. Let $y=W(k, \tau) f$ and $u=y_{\tau}$. We have

$$
i k y_{\tau}=\left[\frac{B}{\tau^{2}}+V\right] y, i k u_{\tau}=\left[\frac{B}{\tau^{2}}+V\right] u+\left[V_{\tau}-2 \frac{B}{\tau^{3}}\right] y
$$

Multiply the first equation by $2 \tau^{-1}$ and add to the second one to get

$$
i k\left[u_{\tau}+2 \frac{u}{\tau}\right]=\left[\frac{B}{\tau^{2}}+V\right] u+\left[V_{\tau}+2 \frac{V}{\tau}\right] y
$$

Let

$$
\phi=\tau^{2} u
$$

Then the equation can be rewritten as

$$
i k \phi_{\tau}=\left[\frac{B}{\tau^{2}}+V\right] \phi+\tau^{2}\left[V_{\tau}+2 \frac{V}{\tau}\right] y, \phi(1)=(B f+V(1) f) /(i k)
$$


Therefore,

$$
\phi(\tau)=U(k, 1, \tau) \phi(1)+\int_{1}^{\tau} s^{2} U(s, \tau)\left[V_{\tau}+2 \frac{V}{s}\right] y d s
$$

Using Conditions $\mathrm{A} 1$ and $\|y\|=\|f\|$, we get an estimate

$$
\|\phi(\tau)\|<C\|f\|_{H^{2}(\Sigma)}+C\|f\| \tau^{2-\gamma}
$$

Thus, (16) yields (10). To prove the estimate on the second derivative in time, we introduce $\psi=y_{\tau \tau}$. Then,

$$
i k \psi_{\tau}=\left[\frac{B}{\tau^{2}}+V\right] \psi+\left[V_{\tau \tau} y+2 V_{\tau} y_{\tau}-4 \frac{B}{\tau^{3}} y_{\tau}+6 \frac{B}{\tau^{4}} y\right]
$$

Multiply the second equation in (15) by $4 \tau^{-1}$ and add to the equation above. We get

$$
i k\left[\psi_{\tau}+4 \frac{\psi}{\tau}\right]=\left[\frac{B}{\tau^{2}}+V\right] \psi+\left[V_{\tau \tau} y+2 V_{\tau} y_{\tau}-2 \frac{B}{\tau^{4}} y+4 \frac{V}{\tau} y_{\tau}+4 \frac{V_{\tau}}{\tau} y\right]
$$

Using the suitable substitution again, one has

$$
\begin{gathered}
\psi(\tau)=\tau^{-4} U(k, 1, \tau) \psi(1) \\
+\tau^{-4} \int_{1}^{\tau} s^{4} U(k, s, \tau)\left[V_{\tau \tau} y+2 V_{\tau} y_{\tau}-2 \frac{B}{s^{4}} y+4 \frac{V}{s} y_{\tau}+4 \frac{V_{\tau}}{s} y\right] d s
\end{gathered}
$$

and

$$
\psi(1)=\left.(i k)^{-2}(B+V)^{2}\right|_{\tau=1} f+(i k)^{-1}\left(V_{\tau}(1)-2 B\right) f
$$

Using estimate on $y_{\tau}$, Conditions A1, and equation (6) to bound $s^{-4} B y$, we get (11).

Differentiate the first equation in (15) in $k$. If $y_{k}=\chi$, then

$$
i k \chi_{\tau}=\left[\frac{B}{\tau^{2}}+V\right] \chi-i y_{\tau}, \chi(1)=0
$$

and

$$
\chi(\tau)=-i \int_{1}^{\tau} U(k, s, \tau) y_{\tau}(s) d s
$$

Now, the estimate (10) yields (12) immediately.

Let us estimate the mixed derivative of $y$, i.e. $\mu=y_{k \tau}$. To do so, differentiate (18) in $\tau$ :

$$
i k \mu_{\tau}=\left[\frac{B}{\tau^{2}}+V\right] \mu+\left[-i y_{\tau \tau}-2 \frac{B}{\tau^{3}} y_{k}+V_{\tau} y_{k}\right]
$$

Multiply (18) by $2 \tau^{-1}$ and add to the last equation. We then have

$$
i k\left[\mu_{\tau}+2 \frac{\mu}{\tau}\right]=\left[\frac{B}{\tau^{2}}+V\right] \mu+\left[-i y_{\tau \tau}+V_{\tau} y_{k}+2 \frac{V}{\tau} y_{k}-2 i \frac{y_{\tau}}{\tau}\right]
$$

which yields

$$
\mu(\tau)=\tau^{-2} \mu(1)+\tau^{-2} \int_{1}^{\tau} s^{2} U(k, s, \tau)\left[-i y_{\tau \tau}+V_{\tau} y_{k}+2 \frac{V}{s} y_{k}-2 i \frac{y_{\tau}}{s}\right] d s
$$

Using estimates on $y_{\tau \tau}, y_{k}, y_{\tau}$ and Conditions A1, we get (13).

The last estimate on $y_{k k}$ can be obtained by differentiating (18) in $k$. If $\eta=y_{k k}$, then

$$
i k \eta_{\tau}=\left[\frac{B}{\tau^{2}}+V\right] \eta-2 i y_{\tau k}, \eta(1)=0
$$


Estimate (13) readily yields (14).

Making some assumptions on angular derivatives of $V$ we obtain quite a different result.

Lemma 1.3. Assume that $V$ is such that

$$
\|V\|_{L^{\infty}(\Sigma)} \in L^{\infty}[1, \infty),\left\|D_{\theta} V(\tau, \theta)\right\|_{L^{\infty}(\Sigma)}<C \tau^{-\gamma},\left\|D_{\theta}^{2} V(\tau, \theta)\right\|_{L^{\infty}(\Sigma)}<C \tau^{1-2 \gamma}
$$

Then, the following asymptotics holds

$$
[W(k, \tau) f](\theta)=\exp \left[(i k)^{-1} \int_{1}^{\tau} V(s, \theta) d s\right] \cdot\left[W_{\text {mod }}(k, \tau) f\right](\theta)
$$

where $W_{\text {mod }}(k, \tau) f \rightarrow W_{\text {mod }}(k, \infty) f$ in $L^{2}(\Sigma)$ as $\tau \rightarrow \infty$ and $f \in L^{2}(\Sigma)$.

Proof. The proof is elementary. Let $y=W(k, \tau) f$. First, let us get an estimate on Dy. We have

$$
i k[D y]_{\tau}=\frac{B}{\tau^{2}}[D y]+V[D y]+y[D V],[D y](1)=D f
$$

and

$$
\|D y\|<C\|f\|_{H^{1}}+C \int_{1}^{\tau} s^{-\gamma} d s<C \tau^{1-\gamma}
$$

Denote

$$
\varphi=(i k)^{-1} \int_{1}^{\tau} V(s, \theta) d s
$$

and write $W(k, \tau) f=\exp (\varphi) \nu(\tau)$. For $\nu$, we have equation

$$
i k \nu_{\tau}=\frac{B}{\tau^{2}} \nu+g, g=\frac{2 D \nu \cdot D \varphi+B \varphi+D \varphi \cdot D \varphi}{\tau^{2}}
$$

and $\nu(1)=f$. But $D \nu=D(y \exp (-\varphi))$. From (19) and (20), we have

$$
\|g\|<C \tau^{-2 \gamma},\|g\| \in L^{1}[1, \infty)
$$

Since $U_{0}(k, 1, \tau) \rightarrow U_{0}(k, 1, \infty)$ strongly, we immediately get the statement of the Lemma.

The meaning of the Lemma is quite simple: if $V(\tau, \theta)$ does not depend much on angle $\theta$, then the $\tau^{-2} B$ term in (6) can be neglected.

The next result in not so surprising in view of recent papers [4, 13.

Lemma 1.4. Assume that $V(\tau)=\tau^{-2}[B Q](\tau)$ and both $V(\tau, \theta)$ and $Q(\tau, \theta)$ satisfy the Conditions $A 1$ with $\gamma>2 / 3$. Then, for any $f \in L^{2}(\Sigma)$, we have

$$
U(k, 1, \tau) f \rightarrow U(k, 1, \infty) f, \quad \tau \rightarrow \infty
$$

Proof. The equation (6) can be rewritten as follows

$$
i k y_{\tau}=\frac{B}{\tau^{2}}[y+Q y]+g, \quad g=-Q \frac{B}{\tau^{2}} y-2 \frac{D Q \cdot D y}{\tau^{2}}
$$

From Lemma 1.2, we get $\left\|\tau^{-2} B y\right\|<C \tau^{-\gamma}$. By interpolation with $\|y\|=\|f\|$, we have $\left\|\tau^{-1} D y\right\|<C \tau^{-\gamma / 2}$. For $Q$, we have $\|Q\|<C \tau^{-\gamma},\left\|\tau^{-2} B Q\right\|<C \tau^{-\gamma}$. Interpolating again, $\left\|\tau^{-1} D Q\right\|<C \tau^{-\gamma}$. Consequently, $\|g\|<C \tau^{-3 \gamma / 2}$ and $\|g\| \in$ $L^{1}[1, \infty)$. Let us consider $z=y+Q y$. For $z$,

$$
i k z_{\tau}=\frac{B}{\tau^{2}} z+g_{1}, g_{1}=g+i k\left(Q y_{\tau}+Q_{\tau} y\right),\left\|g_{1}\right\| \in L^{1}[1, \infty)
$$


Therefore, $z(\tau) \rightarrow z(\infty)$. Since $\|Q(\tau, \theta)\|_{\infty} \rightarrow 0$, the limit of $y$ exists as well.

We believe that the condition $\gamma>2 / 3$ can be replaced by $\gamma>1 / 2$.

The following example is very instructive.

Example. Introduce the spherical coordinates on $\Sigma$ using angles $\phi \in[0,2 \pi), \psi \in$ $[0, \pi)$. Consider, say, diadic decomposition of $[1, \infty)$. For $\tau \in\left[2^{n}, 2^{n+1}\right]$, we let $M(\tau, \phi, \psi)=v(\tau) \sin \left(2^{n} \phi\right) \chi(\psi)$, where $v(\tau)$ is such that $|v(\tau)|<C \tau^{-\gamma},\left|v^{\prime}(\tau)\right|<$ $C \tau^{-1-\gamma},\left|v^{\prime \prime}(\tau)\right|<C \tau^{-1-2 \gamma}$. We also assume $v(\tau)=0$ for $\tau \in\left[2^{n}-1,2^{n}+1\right]$ and any $n$. Function $\chi(\psi)=0$ for $\psi \in[0, \delta] \cup[\pi-\delta, \pi]$ and is infinitely smooth. For $V=M$ and $\gamma>1 / 2$, conditions of Lemma 1.2 are satisfied.

Letting $Q=M$ and $V(\tau, \phi, \psi)=\tau^{-2} B Q(\tau, \phi, \psi)$, we satisfy conditions of the Lemma 1.4 as long as $\gamma>2 / 3$. Therefore, the scalar modification $\exp (\varphi)$ used in Lemma 1.3 is not correct if applied to situation considered in Lemma 1.4 (i.e. the statement of Lemma 1.3 is wrong under conditions of Lemma 1.4). That can be easily proved by contradiction if $v(\tau)$ is chosen properly.

It is important to emphasize that Lemmas 1.1 and 1.2 never used the special properties of Laplace-Beltrami operator. The other two Lemmas did use the fact that Laplace-Beltrami operator is the second-order differential operator.

\section{Asymptotics of the Schrödinger EVolution}

The main goal of this section is to prove an analog of Theorem 0.1 provided that only the radial derivatives are short-range. We need to introduce some notations

first. Recall the definition of $W(k, \tau)$, formula (9). For any $t>0$, consider the following operator

$$
[\mathcal{E}(t) f](x)=(2 i t)^{-3 / 2} \exp \left[i|x|^{2} /(4 t)\right] \cdot W(|x| / t,|x|)[\hat{f}(|x| /(2 t) \theta)](\hat{x})
$$

acting on $f \in \Omega$. The space $\Omega$ consists of functions $f$ such that $\hat{f} \in C^{\infty}\left(\mathbb{R}^{3}\right)$, $\hat{f}$ is compactly supported and vanishes in a neighborhood of the origin (i.e. $\hat{f} \in$ $C_{0}^{\infty}\left(\mathbb{R}^{3} \backslash\{0\}\right)$. For clarity, we explain the meaning of the third factor

$$
W(|x| / t,|x|)[\hat{f}(|x| /(2 t) \theta)](\hat{x})
$$

in more details. To define this function on the sphere of radius $|x|$, we consider $\hat{f}(|x| /(2 t) \theta)$ as the function on the unit sphere (i.e., $\theta \in \Sigma$ ). Since $\hat{f}$ vanishes near the origin, we do not have any problems with definition at $|x|=0$. Then, we act on this function with the operator $W(k, \tau)$ where $k=|x| / t$ and $\tau=|x|$. The resulting function is taken at the point $\hat{x} \in \Sigma$. This third factor is the WKB correction in the time evolution of $\exp (i t H)$ as $t \rightarrow \infty$. We will need to know some properties of $\mathcal{E}(t)$.

Lemma 2.1. For any $t>0, \mathcal{E}(t)$ can be extended to a unitary linear operator on $L^{2}\left(\mathbb{R}^{3}\right)$.

Proof. Fix $t>0$. Linearity of $\mathcal{E}(t)$ on $\Omega$ is obvious. Let us show that it is an isometry on $\Omega$. We have

$$
\|\mathcal{E}(t)\|_{2}^{2}=\frac{1}{(2 t)^{3}} \int_{0}^{\infty} \rho^{2} \int_{\theta \in \Sigma}|W(\rho / t, \rho)[\hat{f}(\rho /(2 t) \sigma)](\theta)|^{2} d \theta d \rho
$$


Since $W(k, \tau)$ is unitary for any $k$ and $\tau$,

$$
\begin{gathered}
\|\mathcal{E}(t)\|_{2}^{2}=\frac{1}{(2 t)^{3}} \int_{0}^{\infty} \rho^{2} \int_{\sigma \in \Sigma}|\hat{f}(\rho /(2 t) \sigma)|^{2} d \sigma d \rho=\frac{1}{(2 t)^{3}} \int_{\mathbb{R}^{3}}|\hat{f}(x /(2 t))|^{2} d x \\
=\int_{\mathbb{R}^{3}}|\hat{f}(x)|^{2} d x=\|f\|^{2}
\end{gathered}
$$

Since $\Omega$ is dense in $L^{2}\left(\mathbb{R}^{3}\right), \mathcal{E}(t)$ can be extended to $L^{2}\left(\mathbb{R}^{3}\right)$ as an isometry. The adjoint operator can be easily computed

$$
\left[\mathcal{E}^{*}(t) f\right](x)=e^{i \frac{3 \pi}{4}}(2 t)^{3 / 2} \cdot \mathcal{F}^{-1}\left\{\exp \left[-i t|w|^{2}\right] \cdot W^{*}(2|w|, 2|w| t)[f(2 t|w| \sigma)](\hat{w})\right\}
$$

The kernel of $\mathcal{E}^{*}(t)$ is trivial. Therefore, the range of $\mathcal{E}(t)$ is $L^{2}\left(\mathbb{R}^{3}\right)$ and $\mathcal{E}(t)$ is unitary.

For $t<0, \mathcal{E}(t)$ can be defined in the same way. Now we are prepared for the main result of this paper.

Theorem 2.1. Assume that $V$ satisfies Conditions $A$. Then, for any $f \in L^{2}\left(\mathbb{R}^{3}\right)$, the following limits exist

$$
\mathcal{W}_{ \pm} f=\lim _{t \rightarrow \pm \infty} \exp (i H t) \mathcal{E}(t) f
$$

We will call these operators $\mathcal{W}_{ \pm}$the modified wave operators.

Proof. We will consider the case $t \rightarrow+\infty$ only. The other situation is analogous. Since both operators $\exp (i H t)$ and $\mathcal{E}(t)$ are unitary, the limit $\mathcal{W}^{+}$, if exists, is an isometry. Also, it is sufficient to prove existence of $\mathcal{W}^{+} f$ for $f \in \Omega$ because $\bar{\Omega}=L^{2}\left(\mathbb{R}^{3}\right)$. Fix $f \in \Omega$. We will use Cook's method to show existence of the limit. To do that, compute a derivative

$$
\frac{d}{d t}[\exp (i t H) \mathcal{E}(t) f]=\exp (i t H)\left[i H \mathcal{E}(t) f+\frac{d}{d t} \mathcal{E}(t) f\right]
$$

To prove existence of the limit, it is sufficient to show that this derivative has $L^{2}(\Sigma)$-norm in $L^{1}[1, \infty)$. This is equivalent to

$$
\left\|i H \mathcal{E}(t) f+\frac{d}{d t} \mathcal{E}(t) f\right\| \in L^{1}[1, \infty)
$$

Checking the last claim is a straightforward calculation that uses properties of evolution $W(k, \tau)$ studied in the previous section. For simplicity, write $[\mathcal{E}(t) f](x)=$ $\kappa(t, x) \cdot Y(t, x)$, where

$$
\kappa(t, x)=(2 i t)^{-3 / 2} \exp \left[i|x|^{2} /(4 t)\right], \quad Y(t, x)=W(|x| / t,|x|)[\hat{f}(|x| /(2 t) \theta)](\hat{x})
$$

Since

$$
\left(i \frac{\partial}{\partial t}+\Delta\right) \kappa(x, t)=0, t>0
$$

we are left with estimating

$$
\Delta(\kappa Y)+i \frac{d}{d t}(\kappa Y)-V \kappa Y=2 \nabla \kappa \cdot \nabla Y+\kappa \Delta Y+i \kappa \frac{d}{d t} Y-V \kappa Y
$$

Let us compute each derivative and show the cancelation of the main terms. We assumed that $f \in \Omega$ and then $\hat{f}(\omega)$ has support within the spherical layer $0<\delta_{1}<$ $|\omega|<\delta_{2}$. Therefore, $Y(x, t)=0$ for $|x|<2 \delta_{1} t$ and for $|x|>2 \delta_{2} t$.

$$
2 \nabla \kappa \cdot \nabla Y=\kappa \frac{i|x|}{t} \frac{d}{d r} Y=\kappa \frac{i|x|}{t}\left\{t^{-1} W_{k}(|x| / t,|x|)[\hat{f}(|x| /(2 t) \theta)](\hat{x})\right.
$$




$$
\begin{gathered}
\left.+W_{\tau}(|x| / t,|x|)[\hat{f}(|x| /(2 t) \theta)](\hat{x})+(2 t)^{-1} W(|x| / t,|x|)\left[\hat{f}_{r}(|x| /(2 t) \theta)\right](\hat{x})\right\} \\
i \kappa \frac{d}{d t} Y=-i \kappa \frac{|x|}{t^{2}} W_{k}(|x| / t,|x|)[\hat{f}(|x| /(2 t) \theta)](\hat{x}) \\
-i \kappa \frac{|x|}{2 t^{2}} W(|x| / t,|x|)\left[\hat{f}_{r}(|x| /(2 t) \theta)\right](\hat{x})
\end{gathered}
$$

We can write $\Delta$ in the following way

$$
\Delta=\frac{1}{r^{2}} \partial_{r}\left(r^{2} \partial_{r}\right)-\frac{B}{r^{2}}=\partial_{r}^{2}+\frac{2}{r} \partial_{r}-\frac{B}{r^{2}}
$$

The first derivative $d / d r$ was calculated in (21). The second derivative gives

$$
\begin{aligned}
\frac{d^{2} Y}{d r^{2}} & =t^{-2} W_{k k}(|x| / t,|x|)[\hat{f}(|x| /(2 t) \theta)](\hat{x})+2 t^{-1} W_{k \tau}(|x| / t,|x|)[\hat{f}(|x| /(2 t) \theta)](\hat{x}) \\
& +W_{\tau \tau}(|x| / t,|x|)[\hat{f}(|x| /(2 t) \theta)](\hat{x})+t^{-2} W_{k}(|x| / t,|x|)\left[\hat{f}_{r}(|x| /(2 t) \theta)\right](\hat{x}) \\
& +t^{-1} W_{\tau}(|x| / t,|x|)\left[\hat{f}_{r}(|x| /(2 t) \theta)\right](\hat{x})+(2 t)^{-2} W(|x| / t,|x|)\left[\hat{f}_{r r}(|x| /(2 t) \theta)\right](\hat{x})
\end{aligned}
$$

Then, we can do some obvious cancelations and combine these terms as follows

$$
\begin{gathered}
2 \nabla \kappa \cdot \nabla Y+\kappa \Delta Y+i \kappa \frac{d}{d t} Y-V \kappa Y= \\
=\kappa\left\{i \frac{|x|}{t} W_{\tau}(|x| / t,|x|)-\frac{B}{|x|^{2}}-V\right\}\left[\hat{f}_{r}(|x| /(2 t) \theta)\right](\hat{x})+R(x, t)
\end{gathered}
$$

By the definition of evolution equation itself, the first term is identically zero. Now, we just need to show that $\|R(\cdot, t)\| \in L^{1}[1, \infty)$. But $R(x, t)=\kappa\left(d^{2} / d r^{2}+\right.$ $\left.2 r^{-1} d / d r\right) Y(x, t)$ and we have to use formulas above and estimates on the derivatives from Lemma 1.2. It is more convenient to write these bounds in spherical coordinates:

$$
\|R\|^{2}<C t^{-3} \int_{2 \delta_{1} t}^{2 \delta_{2} t} \rho^{2}\left\|\left[d^{2} / d \rho^{2}+2 \rho^{-1} d / d \rho\right] Y(\rho, \theta, t)\right\|_{L^{2}(\theta \in \Sigma)}^{2} d \rho<C t^{-4 \gamma}
$$

where the last estimate follows directly from Lemma 1.2 because $\hat{f}(\omega)$ is infinitely smooth. The constant $C$ does depend on $\delta_{1(2)}$. Since $\gamma>1 / 2$, we have $\|R\| \in$ $L^{1}[1, \infty)$ and that finishes the proof.

Notice that this Theorem is strictly stronger than Theorem 0.1. This is due to Lemmas 1.3. 1.4, and the Example considered at the end of previous section. It says, essentially, that the right WKB correction is provided by solution to the evolution equation (6). We do not see how any further asymptotical expansion of $W(k, \tau)$ can be done (i.e. $W(k, \tau)$ seems to be "thing in itself", e.g. like the function $\left.e^{i x}\right)$. That, apparently, is the key difficulty in this and many related problems.

An important example of $V$ from Theorem 2.1 is provided by the following construction which is similar to Example considered in the previous section. Again, take a diadic decomposition of $[1, \infty)$ and define $V$ in spherical coordinates as follows: $V(\rho, \theta)=v(\rho) \cdot h_{n}(\theta)$ for $\rho \in\left[2^{n}, 2^{n+1}\right]$, where $v(\rho)$ is borrowed from Example and $h_{n}(\theta)$ - smooth function on $\Sigma$, different for each $n$.

Now, let us study operators $\mathcal{W}_{ \pm}$. 
Lemma 2.2. For any bounded measurable function $\Phi(\lambda)$, we have an intertwining property

$$
\Phi(H) \mathcal{W}_{ \pm}=\mathcal{W}_{ \pm} \Phi\left(H_{0}\right)
$$

The subspaces $\mathcal{H}_{ \pm}=\operatorname{Ran} \mathcal{W}_{ \pm}$reduce $H$ and $\left.H\right|_{\mathcal{H}_{ \pm}}$are unitarily equivalent to $H_{0}$.

Proof. Again, we give details for $t \rightarrow+\infty$ only. First, consider $\Phi(\lambda)=\exp (i T \lambda)$ with fixed $T$. Let $f \in \Omega$. Then, (23) is equivalent to

$$
\lim _{t \rightarrow+\infty} \exp (i t H) \mathcal{E}(t-T) f=\lim _{t \rightarrow+\infty} \exp (i t H) \mathcal{E}(t) \exp \left(i T H_{0}\right) f
$$

We have

$$
\begin{gathered}
{[\mathcal{E}(t-T) f](x)=(2 i(t-T))^{-3 / 2} \exp \left[i|x|^{2} /(4 t-4 T)\right] \times} \\
W(|x| /(t-T),|x|)[\hat{f}(|x| /(2 t-2 T) \theta)](\hat{x})
\end{gathered}
$$

and

$$
\begin{gathered}
{\left[\mathcal{E}(t) \exp \left(i T H_{0}\right) f\right](x)=(2 i t)^{-3 / 2} \exp \left[i|x|^{2} /(4 t)+i T|x|^{2} /\left(4 t^{2}\right)\right] \times} \\
W(|x| / t,|x|)[\hat{f}(|x| /(2 t) \theta)](\hat{x})
\end{gathered}
$$

Using the estimate $|x|<a t, a>0$, identity

$$
[W(|x| /(t-T),|x|)-W(|x| / t,|x|)] \hat{f}(|x| /(2 t) \theta)=\int_{|x| / t}^{|x| /(t-T)} W_{k}(\alpha,|x|) \hat{f}(|x| /(2 t) \theta) d \alpha
$$

and the bound (12), we easily get

$$
\left\|\mathcal{E}(t-T) f-\mathcal{E}(t) \exp \left(i T H_{0}\right) f\right\|_{L^{2}\left(\mathbb{R}^{3}\right)} \rightarrow 0
$$

as $t \rightarrow \infty$. If (23) is true for dense set $\Omega$, then it must be true for $L^{2}\left(\mathbb{R}^{3}\right)$ since the operators involved are bounded. Thus, (23) holds for $\Phi(\lambda)=\exp (i T \lambda)$ with any $T$. The standard approximation argument then implies (23) in the general setting. We refer the reader to Theorem 4, p. 69, 15] for details. In particular, $E_{\lambda}(H) \mathcal{W}_{ \pm}=\mathcal{W}_{ \pm} E_{\lambda}\left(H_{0}\right)$ and $\mathcal{W}_{ \pm} \mathcal{D}(H) \subseteq \mathcal{D}\left(H_{0}\right)$. Of course, both $\mathcal{D}(H)$ and $\mathcal{D}\left(H_{0}\right)$ are identical to $H^{2}\left(\mathbb{R}^{3}\right)$.

Taking adjoint of (23), we get

$$
E_{\lambda}\left(H_{0}\right) \mathcal{W}_{ \pm}^{*}=\mathcal{W}_{ \pm}^{*} E_{\lambda}(H)
$$

and therefore $\operatorname{Ker} \mathcal{W}_{ \pm}^{*}$ reduces $E_{\lambda}(H)$. That implies Ran $\mathcal{W}_{ \pm}$reduces $E_{\lambda}(H)$ as well. The rest of the Lemma now follows easily.

The following corollary is straightforward

Corollary 2.1. For any $f=\mathcal{W}_{ \pm} g \in \mathcal{H}$, the following asymptotics holds true

$$
\exp (-i t H) f=\mathcal{E}(t) g+\bar{o}(1)
$$

where $\|\bar{o}(1)\|_{L^{2}\left(\mathbb{R}^{3}\right)} \rightarrow 0$ as $t \rightarrow \pm \infty$. 


\section{REFERENCES}

[1] V.S. Buslaev, V.B. Matveev, Wave operators for the Schrödinger equation with slowly decreasing potential, (Russian. English summary), Teoret. Mat. Fiz., 2, 1970, no. 3, 367-376.

[2] H. Cornean, I. Herbst, C. Gérard, Spiraling attractors and quantum dynamics for a class of long-range magnetic fields, MaPhySto Report 2002-36, to appear in J. Funct. Anal.

[3] S.A. Denisov, Wave propagation through sparse potential barriers, (preprint).

[4] S.A. Denisov, Absolutely continuous spectrum for multidimensional Schrödinger operators, IMRN, 2004, no. 74, 3963-3982.

[5] J. Dereziński, C. Gérard, Scattering theory of classical and quantum $N$-particle systems, Texts and Monographs in Physics, Springer-Verlag, Berlin, 1997.

[6] J. Dereziński, C. Gérard, Long-range scattering in the position representation, J. Math. Phys., 38, 1997, no. 8, 3925-3942.

[7] J. Dollard, Asymptotic convergence and the Coulomb interaction, J. Mathematical Phys., 5, 1964, 729-738.

[8] L. Hörmander, The existence of wave operators in scattering theory, Math. Z., 146, 1976, 69-91.

[9] I. Herbst, E. Skibsted, Quantum scattering for potentials independent of $|x|$ : asymptotic completeness for high and low energies, Comm. Partial Differential Equations, 29, 2004, no. $3-4,547-610$.

[10] T. Ikebe, Y. Saito, Limiting absorption method and absolute continuity for the Schrödinger operator, J. Math. Kyoto Univ., 12, 1972, 513-542.

[11] G. Perelman, Stability of the absolutely continuous spectrum for multidimensional Schrödinger operators, Int. Math. Res. Not., 2005, no. 37, 2289-2313.

[12] B. Perthame, L. Vega, Morrey-Campanato estimates for Helmholtz equations, J. Funct. Anal., 164, 1999, no. 2, 340-355.

[13] O. Safronov, On the a.c. spectrum of multi-dimensional Schrödinger operators with slowly decaying potentials, Comm. Math. Phys., Vol. 254, 2005, no. 2, 361-366.

[14] D. Yafaev, Scattering theory: some old and new problems, Lecture Notes in Mathematics, 1735, Springer-Verlag, Berlin, 2000.

[15] D. Yafaev, Mathematical scattering theory, General theory, Translations of Mathematical Monographs, 105, American Mathematical Society, Providence, RI, 1992

[16] D. Yafaev, Wave operators for Schrödinger equation, Theoret. and Math. Phys., 45, no. 2, 1981, 992-998.

University of Wisconsin-Madison, Mathematics Department, 480 Lincoln Dr., MadiSON WI, 53706-1388, USA e-mail: denissov@math.wisc.edu 